\title{
Effect of Zinc and Microbial Inoculation on Soil Enzyme Activities for Maize (Zea mays L.) in Black Soil
}

\author{
Suganya Ayyar*, Saravanan Appavoo, M. Basker, P. Pandiyarajan and R. Kavimani \\ Department of Soil Science and Agricultural Chemistry, NPRC, Vamban, Tamil Nadu \\ Agricultural University, Coimbatore - 3, India \\ *Corresponding author
}

\section{A B S T R A C T}

\begin{tabular}{|l|}
\hline K e y w or d s \\
Maize, Glomus \\
intraradices (AM), Zinc \\
solubilizing, Bacillus $\mathrm{Sp} .$, \\
$\mathrm{ZnSO}_{4}$, Soil enzymes, \\
$\begin{array}{l}\text { Dehydrogenase, Urease } \\
\text { and Phosphatases }\end{array}$ \\
\hline Article Info \\
\hline $\begin{array}{l}\text { Accepted: } \\
\text { 15 July } 2019 \\
\text { Available Online: } \\
\text { 10 August } 2019\end{array}$ \\
\hline
\end{tabular}

Field experiment was conducted in black soils in order to study the soil enzyme activities viz., Dehydrogenase, Urease and Phosphatases for maize using Arbuscular mycorrhizal (AM) fungi and Zinc Solubilizing Bacteria (ZSB) in combination with graded levels of $\mathrm{ZnSO}_{4}$. Treatment consisted of two factors viz., Microbial inoculation ( $M_{1}$ : control, $M_{2}$ : AM fungi, $M_{3}$ : ZSB and $M_{4}: M_{2}+M_{3}$ ) and graded levels of $\mathrm{ZnSO}_{4}\left(\mathrm{~S}_{1}: 0, \mathrm{~S}_{2}: 12.5, \mathrm{~S}_{3}: 25, \mathrm{~S}_{4}: 37.5, \mathrm{~S}_{5}: 50 \mathrm{~kg} \mathrm{ha}^{-1}\right.$ and $\mathrm{S}_{6}: 0.5 \%$ foliar spray ( 45 and 65 DAS) replicated three times in FRBD. The combination of AM and ZSB had enhanced the soil enzyme activities viz., Dehydrogenase, Urease and Phosphatases at vegetative, tasselling and harvest stage of crop growth. However, the Dehydrogenase, Urease and Phosphatases activity was quantitatively increased during tasselling stage due to microbial population, root activities and root exudates higher during tasselling stage. Increased doses of $\mathrm{ZnSO}_{4}$ application had positive effect on the soil enzyme activities viz., Dehydrogenase, Urease and Phosphatases. Combined application of $\mathrm{AM}$ and $\mathrm{ZSB}$ along with $50 \mathrm{~kg}$ of $\mathrm{ZnSO}_{4} \mathrm{ha}^{-1}$ had recorded the highest soil enzyme activities at vegetative, tasselling and harvest stages. However, the dehydrogenase, urease and harvest stage was slightly increased at tasselling stage than vegetative and harvest stage. The application of graded doses of $\mathrm{ZnSO}_{4}$ in combination with AM and ZSB had also increased the soil enzyme activities viz., Dehydrogenase, Urease and Phosphatases, which in turn increased the fertility status of soil.

\section{Introduction}

Maize (Zea mays L.) is one of the world's most widely grown cereals, having great significance as human food, animal feed and raw material source for large number of industrial products (Gajendra et al., 2015). In India, maize is grown in a wide range of environment, extending from extreme semiarid to sub humid and humid regions. It is grown in about 9.09 $\mathrm{M}$ ha and utilized for versatile purposes. It is a main source of calories and minerals for most rural populations.
Soil enzymes are the mediators and catalysts of biochemical processes important in soil functioning such as nutrient mineralization and cycling, decomposition and formation of soil org anic matter and decomposition of xenobiotics (i.e., pesticides). Specifically, oxidoreductores can provide information on the status of key reactions that participate in rate limiting steps of oxidation - reduction process of organic and inorganic materials in soils (Trasar - cepeeda et al., 2000). Sustainability of agricultural systems has become an important issue all over the world. Many issues of sustainability are related to 
soil quality and its change with time (karlen et al., 1997). Soil biological activities have been suggested as one of the important indicator of soil quality (Dick, 1994). It has been proposed that the microbiological and biochemical status of a soil can be used as an early and sensitive indicator of soil ecological stress or restoration processes in both natural and agroecosystem (Ruf et al., 2003). Among these microbiological and biochemical factors, soil enzymes have been suggested as potential indicator of soil quality due to their biological nature, simple measurement and rapid response to changes in soil management when compared to other biological properties (Ling et al., 2010). Soil enzyme activities are 'sensors' of soil degradation. Since they combine information about microbial status, and also from soil physio - chemical conditions (Aon and Colaneri, 2001).

Soil enzyme activities can be used as potential indicators of nutrient cycling processes and fertility management, particularly in long term organic and conventional farming systems (Fliebach et al., 2007). Numerous studies have been conducted to evaluate the potential use of enzymatic activity as an index of soil productivity or soil fertility (Alef and Nannipieri, 1995).

Dehydrogenase activity (DHA) has been recognized as important biochemical indicators in soil (Ryoichi and Senaratne, 2009). Len hard (1956) introduced the concept of determining the metabolic activity of microorganisms in soil and other habitats by measuring DHA. The activity of the DHA is considered an indicator of the oxidative metabolism in soils and thus of the microbiological activity (Garcia et al., 1997) because it is linked to viable cells. Soil DHA reflects the total range of oxidative activity of soil microflora and, consequently it may be a good indicator of microbiological activity in the soil (Skujin, 1976).
Urea cannot be used in plant metabolism directly. The primary physiological role of urease is to allow the organism to use externally supplied and internally generated urea as a nitrogen source. Urease converts urea to products at a rate of at least $10{ }^{14}$ times faster than the urea spontaneous decomposition rate. Urease catalyses urea hydrolysis, thus making urea $-\mathrm{N}$ available to be assimilated into organic compounds (Hogan et al., 1983). Phosphatases catalyse the hydrolysis of ester - phosphate bonds, leading to the release of phosphate $(\mathrm{P})$, which can be taken up by plants or microorganisms (Moscatelli, 2005). It has been shown that the activities of phosphatases (like those of many hydrolyses) depend on several factors such as soil properties, soil organism interactions, plant cover, leachate inputs, and the presence of inhibitors and activators (Speir and Ross, 1978). Phosphatases in $P$ mineralization in soil and the response of these enzyme activities to change in environmental factors and agricultural management.

\section{Materials and Methods}

\section{Experimental soil}

The field experiment was conducted at Cotton Research Station (TNAU), Perambalur. The soil was clay, deep black in colour belonging to Typic Chromustert (Peelamedu series). The initial soil was moderately alkaline, non saline, medium in organic status and low, low and medium in available $\mathrm{N}, \mathrm{P}$ and $\mathrm{K}$, respectively. With regards to the available micronutrient status of the soil, DTPA-Fe, DTPA-Cu and DTPA-Mn were found to be in sufficient level but the availability of DTPA$\mathrm{Zn}$ was found to be deficient.

\section{Field experiments}

Treatment consisted of two factors viz., microbial inoculation $\left(\mathrm{M}_{1}\right.$ : control, $\mathrm{M}_{2}$ : 
Arbuscular Mycorrhiza fungi, $\mathrm{M}_{3}$ : Zinc Solubilizing Bacteria and $\mathrm{M}_{4}: \mathrm{M}_{2}+\mathrm{M}_{3}$ ) and graded levels of $\mathrm{ZnSO}_{4}\left(\mathrm{~S}_{1}: 0, \mathrm{~S}_{2}: 12.5, \mathrm{~S}_{3}: 25\right.$, $\mathrm{S}_{4}: 37.5, \mathrm{~S}_{5}: 50 \mathrm{Kg} \mathrm{ha}^{-1}$ and $\mathrm{S}_{6}: 0.5 \%$ foliar spray@45 and 65 DAS) replicated three times in FRBD. Seeds of maize hybrids (NK 6240) were sown on the sides of the ridges by adopting a spacing of $60 \times 25 \mathrm{~cm}$ along with vermiculite based mycorrhizal inoculum (Glomus intraradices TNAU-03-08) 2 g per hill at a depth of $5 \mathrm{~cm}$. Zinc solubilizing bacteria was applied at $2 \mathrm{~kg} \mathrm{ha}^{-1}$ after mixing it with $25 \mathrm{~kg}$ each of sand and farm yard manure. The recommended fertilizer for maize viz., 250:75:75 $\mathrm{kg} \mathrm{N}, \mathrm{P}_{2} \mathrm{O}_{5}$ and $\mathrm{K}_{2} \mathrm{O} \mathrm{kg} \mathrm{ha}{ }^{-1}$ was followed. The full dose of $\mathrm{P}$ and $\mathrm{K}$ were applied basally and $\mathrm{N}$ was applied at three splits viz., basal (25\%), vegetative (50\%) and tasselling $(25 \%)$ stages. Calculated quantities of $\mathrm{ZnSO}_{4}$ were applied basally as per the treatment schedule.

\section{Data collection and analysis}

Composite surface $(0-15 \mathrm{~cm})$ soil samples were collected from the site before the experiment and analysed for their physical, physio - chemical and chemical properties. Soil samples were also collected from each of the plots at vegetative $\left(30^{\text {th }}\right.$ DAS $)$, tasselling $\left(60^{\text {th }}\right.$ DAS $)$ and harvesting stage of crop for analyse. The samples were air dried and passed through $2 \mathrm{~mm}$ sieve prior to analysis. The $\mathrm{pH}$ and EC were determined in 1:2.5 (soil: water) suspension using digital $\mathrm{pH}$ meter. Organic carbon (OC) content of soil was determined by chromic acid wet oxidation method (Walkley and Black, 1934); available nitrogen content in soil was determined by alkaline permanganate method (Subbiah and Asija, 1956); available Phosphorus content in soil was estimated using olsen's extraction method (Olsen et al., 1945); available potassium in soil was analysed neutral normal ammonium acetate method as outlined by Jackson (1973); exchangeable calcium and magnesium in soil was determined by versenate titration - neutral normal ammonium acetate extract (Jackson, 1973) and available micronutrient $(\mathrm{Zn}, \mathrm{Cu}, \mathrm{Fe}$ and $\mathrm{Mn}$ ) in soil was estimated by Atomic Absorption Spectrophotometer (AAS) after extracting soil samples with DTPA (Diethelene Triamine Penta Acetic Acid) extractant (Linday and Norwell, 1978).

Data were statistically analysed using ANOVA package method of Gomez and Gomez (1984) and least significant difference (LSD) at $5 \%$ probability level was computed to compare the treatments.

\section{Dehydrogenase}

Soil dehydrogenase activity was determined by the method outlined by Casida et al., (1964). Ten g of soil sample was taken in 50 $\mathrm{mL}$ tubes and it was moistened to 50 per cent of water holding capacity by adding distilled water. Then to each tube, $1 \mathrm{~mL}$ of 1 per cent 2,3,5- triphenyl tetrazolium chloride was added. The contents of each tube were then mixed thoroughly at $37 \pm 1^{\circ} \mathrm{C}$ for 48 hours. After incubation period, $20 \mathrm{~mL}$ of methanol was added and stirred for $5 \mathrm{~min}$. The resultant slurry was filtered through Whatman No 42 filter paper. Filtration was continued till disappearance of red colour in the soil. Then the volume of methanol filtrate was made upto $25 \mathrm{~mL}$ and the intensity of red colour was determined in a spectrophotometer at a wavelength of $485 \mathrm{~nm}$ with methanol as a blank. The dehydrogenase enzyme activity was expressed as ( $\mu \mathrm{g}$ of TPF $\mathrm{g} \mathrm{soil}{ }^{-1}$ day $^{-1}$ ).

\section{Urease}

Ten gram of dry and sieved soil was taken in a $100 \mathrm{ml}$ volumetric flask. To this $1.5 \mathrm{ml}$ of toluene was added, mixed well and incubated for 15 minutes. Then $10 \mathrm{ml}$ of 10 per cent urea solution and $20 \mathrm{ml}$ of citrate buffer were 
added, mixed thoroughly, stoppered and incubated for $3 \mathrm{~h}$ at $37^{0} \mathrm{C}$. Then the volume was made up to $100 \mathrm{ml}$ with distilled water, mixed by shaking immediately. The contents were filtered through Whatman No.1 filter paper and $1 \mathrm{ml}$ of filtrate was pipetted out into $50 \mathrm{ml}$ volumetric flask. To this $9 \mathrm{ml}$ of distilled water, $4 \mathrm{ml}$ of phenate and $3 \mathrm{ml}$ of $\mathrm{NaOCl}$ were added, mixed well and allowed to stand for 20 minutes. The volume was made up to $50 \mathrm{ml}$ and mixed well. The bluish green colour developed was read at $630 \mathrm{~nm}$. Simultaneously a blank was also run. The concentration of urease in the sample was obtained from the standard graph using diammonium sulphate and this enzyme activity was expressed as $\left(\mu \mathrm{g} \mathrm{NH}_{4}-\mathrm{N} \mathrm{g}^{-1}\right.$ of soil $\mathrm{h}^{-1}$ ) (Bremner and Mulvaney, 1978).

\section{Phosphatase}

Five $\mathrm{g}$ of soil sample was taken in boiling tube. To this $10 \mathrm{~mL}$ of distilled water, 0.25 $\mathrm{mL}$ of toluene and $1 \mathrm{~mL}$ of $10 \mathrm{mM}$ para nitro phenyl phosphate (PNP) were added and incubated at room temperature for 1 hour. Then $5 \mathrm{~mL}$ of $0.5 \mathrm{M} \mathrm{CaCl}_{2}$ and $20 \mathrm{~mL}$ of 0.5 $\mathrm{M} \mathrm{NaOH}$ were added. The content was filtered using Whatman No 42 and volume was made up to $50 \mathrm{~mL}$ with distilled water. The intensity of yellow colour developed was read at 420 $\mathrm{nm}$ in spectrophotometer (Varian Cary $50 \mathrm{UV}$ visible spectrophotometer). The activity of phosphatase was calculated using standard graph and this enzyme activity was expressed as ( $\mu \mathrm{g}$ PNP $\mathrm{g}^{-1}$ of soil $\mathrm{h}^{-1}$ ) (Bremner and Tabatabai, 1969).

\section{Results and Discussion}

\section{Dehydrogenase activity}

Microbial inoculation had marked influence on dehydrogenase activity over control in black soil (Figure 1). The highest dehydrogenase activity was recorded for $\mathrm{M}_{4}$
(39.5 $\mu \mathrm{g}^{\mathrm{TPF}} \mathrm{g}^{-1}$ of soil day ${ }^{-1}$ ) followed by $\mathrm{M}_{3}\left(36.4 \mu \mathrm{g}\right.$ TPF $\mathrm{g}^{-1}$ of soil day ${ }^{-1}$ ) and $\mathrm{M}_{2}$ (33.8 $\mu \mathrm{g}_{\mathrm{TPF}} \mathrm{g}^{-1}$ of soil day ${ }^{-1}$ ) over control during vegetative stage (Table 1). Graded doses of $\mathrm{Zn}$ application increased the dehydrogenase activity. The highest dehydrogense activity was noticed for $\mathrm{S}_{5}(50.3$ $\mu \mathrm{g}$ TPF $\mathrm{g}^{-1}$ of soil day $\left.{ }^{-1}\right)$ followed by $\mathrm{S}_{4}(44.1$ $\mu \mathrm{g}$ TPF $\mathrm{g}^{-1}$ of soil day $\left.{ }^{-1}\right), \mathrm{S}_{3}\left(37.3 \mu \mathrm{g}\right.$ TPF $\mathrm{g}^{-1}$ of soil day $\left.{ }^{-1}\right)$ and $S_{2}\left(31.7 \mu \mathrm{g}\right.$ TPF $\mathrm{g}^{-1}$ of soil day $^{-1}$ ) over control. Similar trend was noticed at tasselling and harvest stage. The dehydrogenase activity showed slight increase at tasselling stage than vegetative and harvest stages due to microbial population, root activities and root exudates higher during tasselling stage. Dehydrogenase activity is only present in viable cells; it is thought to reflect the total range of oxidative activity of soil microflora and consequently may be considered to be a good indication of microbial activity. It is understandable that the mycorrhizal fungus inoculated soils had higher organic carbon status and biomass carbon that may have provided adequate $\mathrm{C}$ source for the proliferation of heterotrophic microorganism which eventually increased dehydrogenase activity. Subramanian et al., (2009) found that $\mathrm{Zn}$ nutrition appears to increase dehydrogenases activity of inoculated and uninoculated soils which suggest that $\mathrm{Zn}$ is essential for microbial population.

\section{Urease activity}

Microbial inoculation, $\mathrm{M}_{4}$ had recorded highest urease activity $\left(5.5 \mu \mathrm{g} \mathrm{NH}_{4}-\mathrm{N} \mathrm{g}^{-1}\right.$ of soil $\left.\mathrm{h}^{-1}\right)$ followed by $\mathrm{M}_{3}\left(4.5 \mu \mathrm{g} \mathrm{NH}_{4}-\mathrm{N} \mathrm{g}^{-1}\right.$ of soil $\left.\mathrm{h}^{-1}\right)$ and $\mathrm{M}_{2}\left(4.2 \mu \mathrm{g} \mathrm{NH}_{4}-\mathrm{N} \mathrm{g}^{-1}\right.$ of soil $\mathrm{h}^{-1}$ ) over control (Figure 1). Irrespective of microbial inoculations, $\mathrm{S}_{5}$ had highest urease activity $\left(6.0 \mu \mathrm{g} \mathrm{NH}_{4}-\mathrm{N} \mathrm{g}^{-1}\right.$ of soil $\mathrm{h}^{-1}$ ) followed by $\mathrm{S}_{4}\left(5.5 \mu \mathrm{g} \mathrm{NH} 4-\mathrm{N} \mathrm{g}^{-1}\right.$ of soil $\left.\mathrm{h}^{-1}\right)$, $\mathrm{S}_{3}\left(4.7 \mu \mathrm{g} \mathrm{NH}_{4}-\mathrm{N} \mathrm{g}^{-1}\right.$ of soil $\left.\mathrm{h}^{-1}\right)$ and $\mathrm{S}_{2}(4.2$ $\mu \mathrm{g} \mathrm{NH} 4-\mathrm{N} \mathrm{g}^{-1}$ of soil $\mathrm{h}^{-1}$ ) over control (Table 2). 
Initial soil characters

\begin{tabular}{|c|c|}
\hline Taxonomy & $\begin{array}{c}\text { Typic } \\
\text { Chromustert }\end{array}$ \\
\hline Textural class & Clay \\
\hline pH (1:2.5 soil : water) & 8.34 \\
\hline$E C\left(d S m^{-1}\right)$ & 0.30 \\
\hline Free $\mathrm{CaCO}_{3}\left(\mathrm{~g} \mathrm{~kg}^{-1}\right)$ & 90.4 \\
\hline Organic Carbon $\left(\mathrm{g} \mathrm{kg}^{-1}\right)$ & 5.10 \\
\hline $\operatorname{CEC}\left(\operatorname{c~mol}\left(p^{+}\right) \mathrm{kg}^{-1}\right)$ & 36.2 \\
\hline Alkaline $\mathrm{KMnO}_{4}-\mathrm{N}\left(\mathrm{kg} \mathrm{ha}^{-1}\right)$ & 221 \\
\hline Olsen- $\mathbf{P}\left(\mathrm{kg} \mathrm{ha}^{-1}\right)$ & 20.0 \\
\hline Neutral $\mathrm{N} \mathrm{NH}_{4} \mathrm{Oac}-\mathrm{K}\left(\mathrm{kg} \mathrm{ha}^{-1}\right)$ & 245 \\
\hline DTPA Zn $\left(\mathrm{mg} \mathrm{kg}^{-1}\right)$ & 0.80 \\
\hline DTPA Cu (mg kg-1) & 2.52 \\
\hline DTPA Fe $\left(\mathrm{mg} \mathrm{kg}^{-1}\right)$ & 10.2 \\
\hline DTPA Mn $\left(\mathrm{mg} \mathrm{kg}^{-1}\right)$ & 3.45 \\
\hline
\end{tabular}

Fig.1 AMF and ZSB on soil enzyme activities

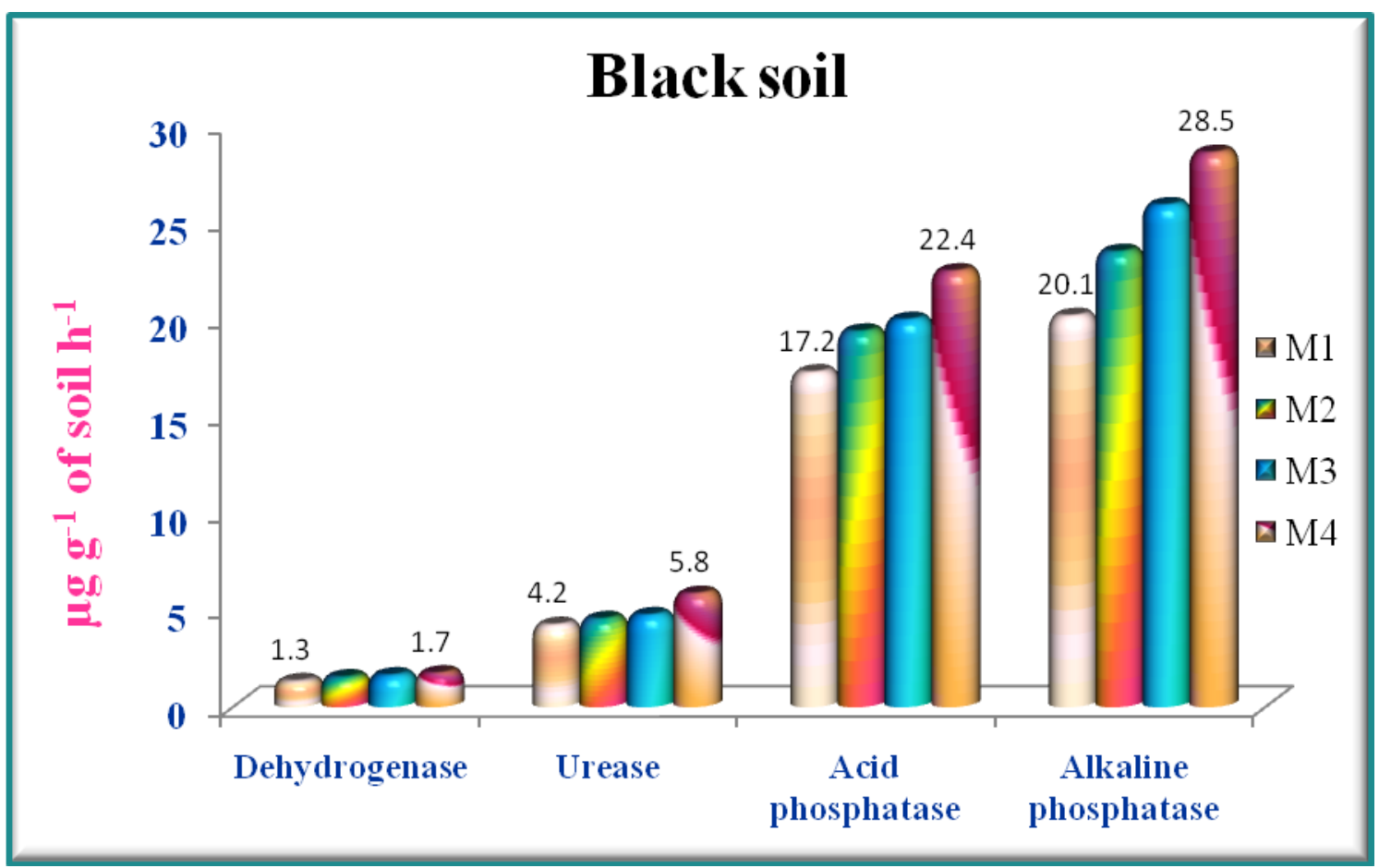


Table.1 Graded levels of Zn with Arbuscular Mycorrhizal Fungi and Zinc Solubilizing Bacteria on soil dehyrogenase activity ( $\mu \mathrm{g}$ TPF $\mathrm{g}^{-1}$ of soil day ${ }^{-1}$ )

\begin{tabular}{|c|c|c|c|c|c|c|c|c|c|c|c|c|c|c|}
\hline \multirow[t]{2}{*}{ Treatments } & \multicolumn{7}{|c|}{ Vegetative stage } & \multicolumn{7}{|c|}{ Tasselling stage } \\
\hline & $\mathbf{S}_{1}$ & $\mathbf{S}_{2}$ & $\mathbf{S}_{\mathbf{3}}$ & $\mathbf{S}_{4}$ & $\mathbf{S}_{5}$ & $S_{6}$ & Mean & $\mathbf{S}_{1}$ & $\mathbf{S}_{\mathbf{2}}$ & $\mathbf{S}_{\mathbf{3}}$ & $\mathbf{S}_{4}$ & $\mathbf{S}_{5}$ & $\mathbf{S}_{6}$ & Mean \\
\hline $\mathbf{M}_{1}$ & 17.3 & 26.4 & 32.6 & 35.2 & 42.8 & 17.5 & 28.6 & 18.7 & 27.4 & 34.7 & 37.4 & 44.8 & 18.8 & 30.3 \\
\hline $\mathbf{M}_{2}$ & 22.0 & 31.3 & 35.6 & 44.5 & 47.2 & 22.0 & 33.8 & 22.8 & 32.6 & 37.1 & 45.4 & 48.0 & 22.9 & 34.8 \\
\hline $\mathbf{M}_{3}$ & 23.0 & 34.2 & 39.6 & 46.1 & 52.3 & 23.1 & 36.4 & 23.1 & 35.0 & 41.5 & 46.9 & 53.2 & 23.2 & 37.2 \\
\hline $\mathbf{M}_{4}$ & 26.0 & 34.7 & 41.2 & 50.4 & 58.9 & 26.0 & 39.5 & 26.8 & 35.2 & 41.7 & 51.0 & 59.4 & 26.6 & 40.1 \\
\hline \multirow[t]{2}{*}{ Mean } & 22.1 & 31.7 & 37.3 & 44.1 & 50.3 & 22.2 & 34.6 & 22.9 & 32.6 & 38.8 & 45.2 & 51.4 & 22.9 & 35.6 \\
\hline & & SEd & & \multicolumn{3}{|c|}{$\mathrm{CD}(0.05)$} & & & SEd & & \multicolumn{3}{|c|}{$\mathrm{CD}(0.05)$} & \\
\hline $\mathbf{M}$ & & 0.67 & & \multicolumn{3}{|c|}{1.4} & & & 0.68 & & \multicolumn{3}{|c|}{1.4} & \\
\hline $\mathbf{S}$ & & 0.82 & & \multicolumn{3}{|c|}{1.7} & & & 0.82 & & \multicolumn{3}{|c|}{1.7} & \\
\hline M X S & & 1.64 & & \multicolumn{3}{|c|}{3.4} & & & 1.63 & & \multicolumn{3}{|c|}{3.4} & \\
\hline
\end{tabular}

\begin{tabular}{|c|c|c|c|c|c|c|c|}
\hline \multirow[t]{2}{*}{ Treatments } & \multicolumn{7}{|c|}{ Harvest stage } \\
\hline & $\mathbf{S}_{1}$ & $\mathbf{S}_{2}$ & $\mathbf{S}_{\mathbf{3}}$ & $\mathbf{S}_{4}$ & $\mathbf{S}_{5}$ & $S_{6}$ & Mean \\
\hline $\mathbf{M}_{1}$ & 16.4 & 25.4 & 31.9 & 34.7 & 41.6 & 16.5 & 27.8 \\
\hline $\mathbf{M}_{2}$ & 21.5 & 30.4 & 34.7 & 43.3 & 46.7 & 21.9 & 33.1 \\
\hline $\mathbf{M}_{3}$ & 23.1 & 33.6 & 38.2 & 46.0 & 51.8 & 23.1 & 36.0 \\
\hline $\mathbf{M}_{4}$ & 25.5 & 34.0 & 40.9 & 48.4 & 58.1 & 25.6 & 38.8 \\
\hline \multirow[t]{2}{*}{ Mean } & 21.6 & 30.9 & 36.4 & 43.1 & 49.6 & 21.8 & 33.9 \\
\hline & & SEd & & \multicolumn{3}{|c|}{$\mathrm{CD}(0.05)$} & \\
\hline M & & 0.65 & & \multicolumn{3}{|c|}{1.4} & \\
\hline $\mathbf{S}$ & & 0.79 & & \multicolumn{3}{|c|}{1.7} & \\
\hline MX S & & 1.59 & & \multicolumn{3}{|c|}{3.3} & \\
\hline
\end{tabular}


Table.2 Graded levels of $\mathrm{Zn}$ with Arbuscular Mycorrhizal fungi and zinc solubilizing bacteria on soil urease activity $\left(\mu \mathrm{g} \mathrm{NH} 4-\mathrm{N} \mathrm{g}^{-1}\right.$ of soil h$\left.{ }^{-1}\right)$

\begin{tabular}{|c|c|c|c|c|c|c|c|c|c|c|c|c|c|c|}
\hline \multirow[t]{2}{*}{ Treatments } & \multicolumn{7}{|c|}{ Vegetative stage } & \multicolumn{7}{|c|}{ Tasselling stage } \\
\hline & $\mathbf{S}_{1}$ & $\mathbf{S}_{2}$ & $\mathbf{S}_{3}$ & $\mathbf{S}_{4}$ & $\mathbf{S}_{5}$ & $\mathbf{S}_{6}$ & Mean & $\mathbf{S}_{1}$ & $\mathbf{S}_{2}$ & $\mathbf{S}_{\mathbf{3}}$ & $\mathbf{S}_{4}$ & $\mathbf{S}_{5}$ & $\mathbf{S}_{6}$ & Mean \\
\hline $\mathbf{M}_{1}$ & 3.1 & 3.6 & 4.1 & 4.7 & 5.1 & 3.0 & 3.9 & 3.3 & 3.8 & 4.4 & 4.9 & 5.3 & 3.2 & 4.2 \\
\hline $\mathbf{M}_{2}$ & 3.3 & 3.8 & 4.3 & 5.0 & 5.6 & 3.3 & 4.2 & 3.5 & 4.0 & 4.6 & 5.3 & 5.8 & 3.5 & 4.5 \\
\hline $\mathbf{M}_{3}$ & 3.5 & 4.1 & 4.6 & 5.4 & 5.9 & 3.5 & 4.5 & 3.7 & 4.3 & 4.9 & 5.6 & 6.1 & 3.7 & 4.7 \\
\hline $\mathbf{M}_{4}$ & 4.0 & 5.1 & 5.7 & 6.8 & 7.3 & 4.0 & 5.5 & 4.2 & 5.3 & 6.1 & 7.2 & 7.8 & 4.2 & 5.8 \\
\hline \multirow[t]{2}{*}{ Mean } & 3.5 & 4.2 & 4.7 & 5.5 & 6.0 & 3.5 & 4.5 & 3.7 & 4.4 & 5.0 & 5.8 & 6.3 & 3.7 & 4.8 \\
\hline & & SEd & & \multicolumn{3}{|c|}{$\mathrm{CD}(0.05)$} & & & SEd & & \multicolumn{3}{|c|}{$\mathrm{CD}(0.05)$} & \\
\hline $\mathbf{M}$ & & 0.03 & & \multicolumn{3}{|c|}{0.1} & & & 0.07 & & \multicolumn{3}{|c|}{0.1} & \\
\hline $\mathbf{S}$ & & 0.04 & & \multicolumn{3}{|c|}{0.1} & & & 0.08 & & \multicolumn{3}{|c|}{0.1} & \\
\hline M X S & & 0.08 & & \multicolumn{3}{|c|}{0.2} & & & 0.05 & & \multicolumn{3}{|c|}{0.1} & \\
\hline
\end{tabular}

\begin{tabular}{|c|c|c|c|c|c|c|c|}
\hline \multirow{2}{*}{ Treatments } & \multicolumn{9}{|c|}{ Harvest stage } \\
\cline { 2 - 8 } & $\mathbf{S}_{\mathbf{1}}$ & $\mathbf{S}_{\mathbf{2}}$ & $\mathbf{S}_{\mathbf{3}}$ & $\mathbf{S}_{\mathbf{4}}$ & $\mathbf{S}_{\mathbf{5}}$ & $\mathbf{S}_{\mathbf{6}}$ & Mean \\
\hline $\mathbf{M}_{\mathbf{1}}$ & 2.9 & 3.5 & 3.8 & 4.4 & 4.9 & 2.8 & $\mathbf{3 . 7}$ \\
\hline $\mathbf{M}_{\mathbf{2}}$ & 3.0 & 3.4 & 4.0 & 4.6 & 5.1 & 3.0 & $\mathbf{3 . 9}$ \\
\hline $\mathbf{M}_{\mathbf{3}}$ & 3.1 & 3.7 & 4.2 & 5.0 & 5.4 & 3.1 & $\mathbf{4 . 1}$ \\
\hline $\mathbf{M}_{\mathbf{4}}$ & 3.7 & 4.7 & 5.2 & 6.3 & 6.7 & 3.6 & $\mathbf{5 . 0}$ \\
\hline Mean & $\mathbf{3 . 2}$ & $\mathbf{3 . 8}$ & $\mathbf{4 . 3}$ & $\mathbf{5 . 1}$ & $\mathbf{5 . 5}$ & $\mathbf{3 . 1}$ & $\mathbf{4 . 2}$ \\
\hline & & $\mathrm{SEd}$ & & & $\mathrm{CD}(0.05)$ & & \\
\hline $\mathbf{M}$ & & 0.03 & & & 0.1 & & \\
\hline $\mathbf{S}$ & & 0.03 & & & 0.1 & & \\
\hline $\mathbf{M} \mathbf{X}$ & & 0.06 & & \multicolumn{3}{|c|}{0.1} \\
\hline
\end{tabular}


Table.3 Graded levels of $\mathrm{Zn}$ with Arbuscular Mycorrhizal fungi and zinc solubilizing bacteria on soil acid phosphatase activity $\left(\mu \mathrm{g}\right.$ p-nitrophenol $\mathrm{g}^{-1}$ of soil $\mathrm{h}^{-1}$ )

\begin{tabular}{|c|c|c|c|c|c|c|c|c|c|c|c|c|c|c|}
\hline \multirow[t]{2}{*}{ Treatments } & \multicolumn{7}{|c|}{ Vegetative stage } & \multicolumn{7}{|c|}{ Tasselling stage } \\
\hline & $\mathbf{S}_{1}$ & $\mathbf{S}_{2}$ & $\mathbf{S}_{\mathbf{3}}$ & $\mathbf{S}_{4}$ & $\mathbf{S}_{5}$ & $\mathbf{S}_{6}$ & Mean & $\mathbf{S}_{1}$ & $\mathbf{S}_{2}$ & $\mathbf{S}_{\mathbf{3}}$ & $\mathbf{S}_{4}$ & $\mathbf{S}_{5}$ & $\mathbf{S}_{6}$ & Mean \\
\hline $\mathbf{M}_{1}$ & 15.0 & 16.2 & 16.5 & 17.9 & 19.0 & 15.0 & 16.6 & 15.3 & 16.4 & 17.6 & 18.9 & 19.4 & 15.3 & 17.2 \\
\hline $\mathbf{M}_{2}$ & 16.0 & 17.9 & 19.1 & 20.2 & 21.8 & 16.0 & 18.5 & 16.5 & 18.6 & 19.7 & 21.0 & 22.5 & 17.5 & 19.3 \\
\hline $\mathbf{M}_{3}$ & 17.3 & 18.2 & 19.0 & 20.8 & 21.7 & 17.3 & 19.1 & 18.1 & 19.0 & 19.9 & 21.4 & 22.7 & 18.0 & 19.9 \\
\hline $\mathbf{M}_{4}$ & 17.6 & 20.0 & 23.5 & 24.2 & 25.3 & 17.7 & 21.4 & 18.1 & 21.7 & 24.7 & 25.0 & 26.5 & 18.3 & 22.4 \\
\hline \multirow[t]{2}{*}{ Mean } & 16.5 & 18.1 & 19.5 & 20.8 & 22.0 & 16.5 & 18.9 & 17.0 & 18.9 & 20.5 & 21.6 & 22.8 & 17.3 & 19.7 \\
\hline & & SEd & & \multicolumn{3}{|c|}{$\mathrm{CD}(0.05)$} & & & SEd & & \multicolumn{3}{|c|}{$\mathrm{CD}(0.05)$} & \\
\hline M & & 0.36 & & \multicolumn{3}{|c|}{0.8} & & & 0.38 & & \multicolumn{3}{|c|}{0.8} & \\
\hline $\mathbf{S}$ & & 0.43 & & \multicolumn{3}{|c|}{0.9} & & & 0.46 & & \multicolumn{3}{|c|}{1.0} & \\
\hline MXS & & 0.86 & & \multicolumn{3}{|c|}{1.8} & & & 0.88 & & \multicolumn{3}{|c|}{1.8} & \\
\hline
\end{tabular}

\begin{tabular}{|c|c|c|c|c|c|c|c|}
\hline \multirow[t]{2}{*}{ Treatments } & \multicolumn{7}{|c|}{ Harvest stage } \\
\hline & $\mathbf{S}_{1}$ & $\mathbf{S}_{2}$ & $\mathbf{S}_{\mathbf{3}}$ & $\mathbf{S}_{4}$ & $\mathbf{S}_{5}$ & $S_{6}$ & Mean \\
\hline $\mathbf{M}_{1}$ & 14.8 & 16.0 & 16.2 & 17.3 & 18.9 & 14.8 & 16.3 \\
\hline $\mathbf{M}_{2}$ & 15.2 & 17.5 & 18.3 & 19.9 & 21.2 & 15.3 & 17.9 \\
\hline $\mathbf{M}_{3}$ & 16.8 & 17.7 & 18.5 & 20.6 & 21.1 & 16.9 & 18.6 \\
\hline $\mathbf{M}_{4}$ & 17.4 & 19.9 & 22.8 & 23.6 & 24.9 & 17.4 & 21.0 \\
\hline \multirow[t]{2}{*}{ Mean } & 16.1 & 17.8 & 19.0 & 20.4 & 21.5 & 16.1 & 18.5 \\
\hline & & SEd & \multicolumn{4}{|c|}{$\mathrm{CD}(0.05)$} & \\
\hline M & & 0.35 & & \multicolumn{3}{|c|}{0.7} & \\
\hline $\mathbf{S}$ & & 0.42 & & \multicolumn{3}{|c|}{0.9} & \\
\hline MX S & & 0.85 & & \multicolumn{3}{|c|}{1.8} & \\
\hline
\end{tabular}


Table.4 Graded levels of Zn with Arbuscular Mycorrhizal Fungi and Zinc solubilizing bacteria on soil alkaline phosphatase activity ( $\mu \mathrm{g}$ p-nitrophenol $\mathrm{g}^{-1}$ of soil $^{-1}$ )

\begin{tabular}{|c|c|c|c|c|c|c|c|c|c|c|c|c|c|c|}
\hline \multirow[t]{2}{*}{ Treatments } & \multicolumn{7}{|c|}{ Vegetative stage } & \multicolumn{7}{|c|}{ Tasselling stage } \\
\hline & $\mathbf{S}_{1}$ & $\mathbf{S}_{2}$ & $\mathbf{S}_{\mathbf{3}}$ & $\mathbf{S}_{4}$ & $\mathbf{S}_{5}$ & $\mathbf{S}_{6}$ & Mean & $\mathbf{S}_{1}$ & $\mathbf{S}_{\mathbf{2}}$ & $\mathbf{S}_{\mathbf{3}}$ & $\mathbf{S}_{4}$ & $\mathbf{S}_{5}$ & $\mathbf{S}_{6}$ & Mean \\
\hline $\mathbf{M}_{1}$ & 15.2 & 17.8 & 18.3 & 19.4 & 22.9 & 15.2 & 18.1 & 17.1 & 19.3 & 20.5 & 22.6 & 23.8 & 17.2 & 20.1 \\
\hline $\mathbf{M}_{2}$ & 19.0 & 21.3 & 22.1 & 24.5 & 26.9 & 19.0 & 22.1 & 19.8 & 23.1 & 24.9 & 25.8 & 27.1 & 19.8 & 23.4 \\
\hline $\mathbf{M}_{3}$ & 19.4 & 21.4 & 25.2 & 27.3 & 29.6 & 19.4 & 23.7 & 20.0 & 24.8 & 26.3 & 30.5 & 32.9 & 20.1 & 25.8 \\
\hline $\mathbf{M}_{4}$ & 22.0 & 23.9 & 26.3 & 30.1 & 33.2 & 22.1 & 26.3 & 22.9 & 27.4 & 29.5 & 32.6 & 35.5 & 23.0 & 28.5 \\
\hline \multirow[t]{2}{*}{ Mean } & 18.9 & 21.1 & 23.0 & 25.3 & 28.2 & 18.9 & 22.6 & 20.0 & 23.7 & 25.3 & 27.9 & 29.8 & 20.0 & 24.4 \\
\hline & & SEd & & \multicolumn{3}{|c|}{$\mathrm{CD}(0.05)$} & & & SEd & & \multicolumn{3}{|c|}{$\mathrm{CD}(0.05)$} & \\
\hline $\mathbf{M}$ & & 0.43 & & \multicolumn{3}{|c|}{0.9} & & & 0.47 & & \multicolumn{3}{|c|}{1.0} & \\
\hline $\mathbf{S}$ & & 0.51 & & \multicolumn{3}{|c|}{1.1} & & & 0.56 & & \multicolumn{3}{|c|}{1.2} & \\
\hline MX S & & 1.02 & & \multicolumn{3}{|c|}{2.1} & & & 1.12 & & \multicolumn{3}{|c|}{2.3} & \\
\hline
\end{tabular}

\begin{tabular}{|c|c|c|c|c|c|c|c|}
\hline \multirow[t]{2}{*}{ Treatments } & \multicolumn{7}{|c|}{ Harvest stage } \\
\hline & $\mathbf{S}_{1}$ & $\mathbf{S}_{\mathbf{2}}$ & $\mathbf{S}_{\mathbf{3}}$ & $\mathbf{S}_{4}$ & $\mathbf{S}_{5}$ & $\mathbf{S}_{6}$ & Mean \\
\hline $\mathbf{M}_{1}$ & 14.5 & 17.0 & 17.4 & 20.4 & 22.9 & 14.6 & 17.8 \\
\hline $\mathbf{M}_{2}$ & 18.7 & 20.4 & 21.9 & 24.0 & 26.8 & 18.7 & 21.8 \\
\hline $\mathbf{M}_{3}$ & 19.0 & 21.1 & 24.3 & 26.2 & 28.5 & 19.0 & 23.0 \\
\hline $\mathbf{M}_{4}$ & 21.8 & 23.2 & 25.9 & 29.2 & 32.5 & 21.8 & 25.7 \\
\hline \multirow[t]{2}{*}{ Mean } & 18.5 & 20.4 & 22.4 & 25.0 & 27.7 & 18.5 & 22.1 \\
\hline & & SEd & & \multicolumn{3}{|c|}{$\mathrm{CD}(0.05)$} & \\
\hline $\mathbf{M}$ & & 0.42 & & \multicolumn{3}{|c|}{0.9} & \\
\hline $\mathbf{S}$ & & 0.50 & & \multicolumn{3}{|c|}{1.0} & \\
\hline MX S & & 1.03 & & \multicolumn{3}{|c|}{2.2} & \\
\hline
\end{tabular}


The trend of results obtained during tasselling and harvest stages of crop growth on urease activity was similar to that of vegetative stage. However, the urease activity was quantitatively increased during tasselling stage probably due to higher amount of root exudates which lead to higher microbial population resulting in increased urease activity. The change in microbial community and release of root exudates as a result of mycorrhizal colonization modified the soil enzymes activities.

\section{Phosphatase activity}

During vegetative stage, among the microbial inoculations, $\mathrm{M}_{4}$ had registered highest acid and alkaline phosphatase were 21.4 and 26.3 $\mu \mathrm{g}$ p-nitrophenol $\mathrm{g}^{-1}$ of soil $\mathrm{h}^{-1}$ followed by $\mathrm{M}_{3}$ (19.1 and $23.7 \mu \mathrm{g}$ p-nitrophenol $\mathrm{g}^{-1}$ of soil $\left.\mathrm{h}^{-1}\right)$ and $\mathrm{M}_{2}(18.5$ and $22.1 \mu \mathrm{g}$ p-nitrophenol $\mathrm{g}$ ${ }^{1}$ of soil $\mathrm{h}^{-1}$ ) over control (Figure 1). Among the graded doses of $\mathrm{ZnSO}_{4}$ application $\mathrm{S}_{5}$ had highest acid and alkaline phosphatase (22.0 and $28.2 \mu \mathrm{g}$ p-nitrophenol $\mathrm{g}^{-1}$ of soil $\mathrm{h}^{-1}$, respectively) followed by $S_{4}, S_{3}$ and $S_{2}$ over control. Similar trend was noticed at tasselling and harvest stage (Table 3 and 4). However, the tasselling stage, acid and alkaline phosphatase was slightly higher which may probably due to plant metabolic and root activities. Soil phosphatase plays on important role in the $\mathrm{P}$ nutrition of plants because it mediates the release of inorganic phosphorus from organically bound phosphorus. Phosphatase enzymes are also directly involved in the acquisition of phosphorus by plants.

Mycorrhizal colonization and ZSB viz., Bacillus sp. has been shown to influence phosphatase activity. Subramanian et al., (2008) have shown that the soil phosphatase activity in the maize rhizosphere increased by 2-3 times as that of uninoculated soil suggesting that this could reduce the $\mathrm{pH}$ and stimulate the availability of $\mathrm{P}$.

\section{References}

Alef, K., Nannipieri, P., 1995. Methods in Applied soil microbiology and biochemistry. Academic Press, London.

Aon, M. A., Colaneri, A.C., 2001. Temporal and Spatial evolution of enzyme activities and physio - chemical properties in an agricultural soil. Applied soil Ecology 18, 255-270.

Bremner, J.M and M.A. Tabatabai. 1969. Use of $\rho$-nitrophenyl phosphate for assay of soil phosphatase activity. Soil Biol. Biochem., 1: 301-307.

Bremner, J.M. and R.L. Mulvaney. 1978. Urease activity in soils. In: Soil Enzymes (ed. R.G. Burns), Academic Press, London. pp. 149196.

Casida, C.E.J., D.A. Klien and T. Santro. 1964. Soil dehydrogenace activity. Soil Sci., 98: 370-376.

Dick, R. P., 1994. Soil enzyme activities as indicators of soil quality. In: Doran, J, W., Coleman, D. C., Bezdicek, D. F., Stewart, B. A. (Eds.), Defining soil for a sustainable environment. SSSA Spec. Pub. SSSA, Madison, WI.

Fliebach, A., Oberholzer, H. R., Gunst, L., Mader, P., 2007. Soil organic matter and biological soil quality indicators after 21 years of organic and conventional farming. Agriculture, Ecosystems and Environment $118,273-284$.

Garcia, C., hemandez. T., Costa, F., 1997. Potential use of dehydrogenase activity as an index of microbial activity in degradation soils. Communications in soil science and plant analysis.

Gomez, K.A. and A. A. Gomez. 1984. Statistical Procedures for Agricultural Research, Pub: John Wiley and Sons, New Delhi. p 680.

Hogan, M. E., Swift, I. E. and Done, J. (1983). Urease assay and ammonia release from leaf tissue. phytochemisrty, 22(3), 663-667.

Jackson, M.L. 1973. Soil chemical analysis. Prentice Hall of India Private Ltd., New Delhi, pp. 56-70.

Karlen, D, L., Mausbach, M. J and Doran, J. W. 1997. Soil Quality: a concept, definition and frame work for evaluation. Soil science society of America journal 61, 4-10. 
Len hard, G., 1956. The dehydrogenase activity in soil as a measure of the activity of soil microorganisms. Z Pflanzenemaenr Dueng Bodenked 73, 1-11.

Lindsay, W.L. and W.A. Norvell. 1978. Development of DTPA soil test for zinc, iron, manganese and copper. Soil Sci. Soc. Am. J., 42: 421-428.

Ling, D.J., et al., 2010. Impacts of simulated acid rain on soil enzyme activities in a latosol. Ecotoxicology and Environmental Safty. http;//dx.doi.org/10.1016/j.ecoenv.2010.07. 024.

Moscalelli, M. C., Lagomarsino, A., De Angelis, O and Grego, S. (2005). Seasonality of soil biological properties in a popular plantation growing under elevated atmospheric $\mathrm{CO}_{2}$. Appl Soil Ecol 30: 162-173.

Olsen, S.R., C.V. Cole, F.S. Watenabe and L.A. Dean. 1954. Estimation of available phosphorus in soils by extraction with sodium bicarbonate. U.S.D.A. Circ. 939, U.S. Govt. Printing Office, Washington, DC.

Ruf, A., Beck, I., Drener, P., Hund - Rinke, K., Rombke, J., Spelda, J., 2003. A biological classification concept for the assessment of soil quality, biological classification scheme (BBK), Agriculture, Ecosystems and Environment 98, 263-271.

Ryoichi, D., Senaratne, L. R., 2009. Soil dehydrogenase in a land degradation rehabilitation gradient: observations from a savanna site with a wet/dry seasonal cycle. Revista De Biologica Tropical 57, 223-234.
Skujins, J., 1976. Enzymes in soil. In: Mc Laren AD., Pelerson, G. H. (Eds.), Soil Biochemistry, Marcel pekker, Inc. New York USA, pp.371-414.

Speir, T. W., Ross, D.J. (1978). Soil phosphatase and sulphatase. In: Burns R.G (Ed) soil enzymes. Academic, London, pp 197-250.

Subbiah, B.V. and G.C. Asija. 1956. A rapid procedure for estimation of available nutrients in soils. Curr. Sci., 25: 259 - 260.

Subramanian, K.S., C. Bharathi and A. Jegan. 2008. Response of maize to mycorrhizal colonization at varying levels of zinc and phosphorus. Biol. Fertil. Soils, 45:133-144.

Subramanian, K.S., V. Tenshia, K. Jayalakshmi and V. Ramachandran. 2009. Biochemical changes and zinc fractions in arbuscular mycorrhizal fungus (Glomus intraradices) inoculated and uninoculated soils under differential zinc fertilization. Appl. Soil Ecol., 43: 32-39.

Trasar - Cepeda, C., Leiros, M. C and F. GilSotres, 2000. Biochemical properties of acid soils under climate vegetation (Atlantic oakwood) in an area of the European temperate- humid zone (Galica/NW Spain): Specific Parameters. Soil Bio. Biochem, 32(6): 747-755.

Walkley, A and C.A. Black. 1934. An examination of the Deglgareff method for determining soil organic matter and proposed modification of chromic acid titration method. Soil Sci., 37: 29 - 38.

\section{How to cite this article:}

Suganya Ayyar, Saravanan Appavoo, M. Basker, P. Pandiyarajan and Kavimani, R. 2019. Effect of Zinc and Microbial Inoculation on Soil Enzyme Activities for Maize (Zea mays L.) in Black soil. Int.J.Curr.Microbiol.App.Sci. 8(08): 1804-1814. doi: https://doi.org/10.20546/ijcmas.2019.808.213 